\title{
Oxygen Ion Implantation Isolation Planar Process for AlGaN/GaN HEMTs
}

\author{
Jin-Yu Shiu, Jui-Chien Huang, Vincent Desmaris, Chia-Ta Chang, Chung-Yu Lu, Kazuhide Kumakura, \\ Toshiki Makimoto, Herbert Zirath, Member, IEEE, Niklas Rorsman, and Edward Yi Chang, Senior Member, IEEE
}

\begin{abstract}
A multienergy oxygen ion implantation process was demonstrated to be compatible with the processing of highpower microwave AlGaN/GaN high electron mobility transistors (HEMTs). HEMTs that are isolated by this process exhibited gate-lag- and drain-lag-free operation. A maximum output power density of $5.3 \mathrm{~W} / \mathrm{mm}$ at $V_{\mathrm{gs}}=-4 \mathrm{~V}$ and $V_{\mathrm{ds}}=50 \mathrm{~V}$ and a maximum power added efficiency of $51.5 \%$ at $V_{\mathrm{gs}}=-4 \mathrm{~V}$ and $V_{\mathrm{ds}}=30 \mathrm{~V}$ at $3 \mathrm{GHz}$ were demonstrated on HEMTs without field plates on sapphire substrate. This isolation process results in planar HEMTs, circumventing potential problems with enhanced gate leakage due to the gate contacting the 2-D electron gas at the mesa sidewall.
\end{abstract}

Index Terms-GaN, high electron mobility transistors (HEMTs), implantation, power density, pulsed $I-V$, transient.

\section{INTRODUCTION}

D EVICE isolation of GaN-based high electron mobility transistors (HEMTs) is conventionally realized by dry etching to define the device active region [1]. Implantation isolation maintains the planarity of the device, which may increase the yield and uniformity in GaN HEMT and monolithic microwave integrated circuit (MMIC) processes.

Implantation isolation have been studied in pure $\mathrm{GaN}$ or AlGaN material using $\mathrm{H}^{+}, \mathrm{He}^{+}, \mathrm{N}^{+}, \mathrm{F}^{+}, \mathrm{Mg}^{+}, \mathrm{Ar}^{+}$, and $\mathrm{Zn}^{+}$ions [2]-[8]. The $\mathrm{O}^{+}$ion implant isolation was also investigated on AlGaAs [9], InAlN [10], and GaN (n-type doping)/GaN materials [11] to study the isolation quality, and $\mathrm{P} / \mathrm{He}, \mathrm{Ar}^{+}$, and $\mathrm{N}^{+}$ions have been employed in $\mathrm{AlGaN} / \mathrm{GaN}$ HEMTs [12]-[14].

In this letter, multiple energy $\mathrm{O}^{+}$ion implantation was applied for isolation in the fabrication of $\mathrm{AlGaN} / \mathrm{GaN}$ HEMTs.

Manuscript received January 22, 2007; revised April 2, 2007. This work was supported in part by the Ministry of Education, by the Ministry of Economic Affairs, and by the National Science Council of China under Contract NSC 942752-E-009-001-PAE and Contract 94-EC-17-A-05-S1-020. The review of this letter was arranged by Editor J. del Alamo.

J.-Y. Shiu and J.-C. Huang are with the Department of Materials Science and Engineering, National Chiao Tung University, Hsinchu 300, Taiwan, R.O.C., and also with Microwave Electronics Laboratory, Microtechnology and Nanoscience (MC2-MEL), Chalmers University of Technology, 41296 Gothenburg, Sweden.

V. Desmaris, H. Zirath, and N. Rorsman are with the Microwave Electronics Laboratory, Microtechnology and Nanoscience (MC2-MEL), Chalmers University of Technology, 41296 Gothenburg, Sweden.

C.-T. Chang, C.-Y. Lu, and E. Y. Chang are with the Department of Materials Science and Engineering, National Chiao Tung University, Hsinchu 300, Taiwan, R.O.C. (e-mail: edc@mail.nctu.edu.tw).

K. Kumakura and T. Makimoto are with the NTT Basic Research Laboratories, NTT Corporation, Atsugi 243-0198, Japan.

Digital Object Identifier 10.1109/LED.2007.896904
The motivations for this are given as follows: First, $\mathrm{O}^{+}$ion isolation implantation has better thermal stability compared to light atomic mass ion (i.e., $\mathrm{H}^{+}$or $\mathrm{He}^{+}$). Second, the lower implantation incident energy decreases the probability of surface damage of the device and increases the yield. Third, multiple incident energy and higher implantation ion density were used to ensure high-quality isolation in both AlGaN Schottky and GaN buffer layers with good thermal stability. The latter motivations are the biggest differences between the isolation tests and real HEMT device processes.

Furthermore, in a mesa isolation process, the 2-D electron gas (2DEG) channel is exposed on the sidewall. The gate metallization contacts the 2DEG, causing an excessive gate leakage current and a degradation of the breakdown voltage of the device [15]. This problem should be absent in an implantation process for isolation. For InGaAs HEMTs, a method of recessing the channel edge into the mesa sidewall with the selective etching characteristics of Schottky and buffer layers was developed [16]. The AlGaN and $\mathrm{GaN}$ materials are chemically stable at room temperature, and no convenient selective wet etch process has been demonstrated.

In this letter, the gate leakages on mesa and implanted HEMTs are compared. The multiple incident energies and dose concentration are optimized to assure process compatibility and minimize surface damage problem, as demonstrated by pulsed current-voltage $(I-V)$ and load-pull measurements on AlGaN/GaN HEMTs.

\section{EXPERIMENT}

$\mathrm{AlGaN} / \mathrm{GaN}$ heterostructures were grown on sapphire by metal organic chemical vapor deposition (MOCVD) by Hitachi Cable Corporation (material 1) and NTT Basic Research Laboratories (material 2). They consist of a 2- $\mu \mathrm{m}$-thick unintentionally doped $\mathrm{GaN}$ buffer layer followed by $30 \mathrm{~nm}$ of an undoped $\mathrm{Al}_{0.3} \mathrm{Ga}_{0.7} \mathrm{~N}$ Schottky layer. From Hall measurements, the sheet carrier concentration and electron mobility of materials 1 and 2 were determined to be $1 \times 10^{13} \mathrm{~cm}^{-2}$ and $900 \mathrm{~cm}^{-2} /(\mathrm{V} \cdot \mathrm{s})$, and $1 \times 10^{13} \mathrm{~cm}^{-2}$ and $1100 \mathrm{~cm}^{-2} /(\mathrm{V} \cdot \mathrm{s})$, respectively.

The photoresist S1818 was used as an implantation mask to define the active region of devices. The HEMT device experiment consists of the following three pieces: one piece of material 1 with $2 \times 50 \times 0.6 \mu \mathrm{m}^{2}$ gates defined in the middle of the 4- $\mu \mathrm{m}$ source-drain spacing and two pieces of material 2 with $2 \times 50 \times 2 \mu \mathrm{m}^{2}$ gates defined in the middle of the $10-\mu \mathrm{m}$ source-drain spacing. The HEMTs were fabricated using an 


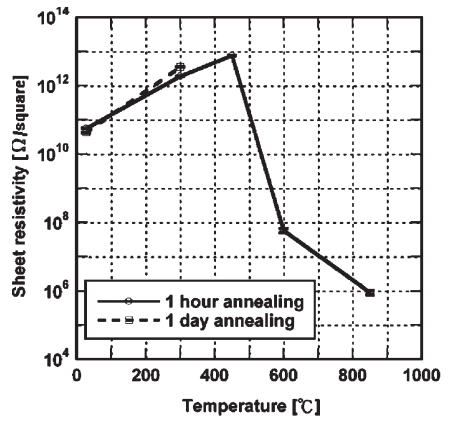

(a)

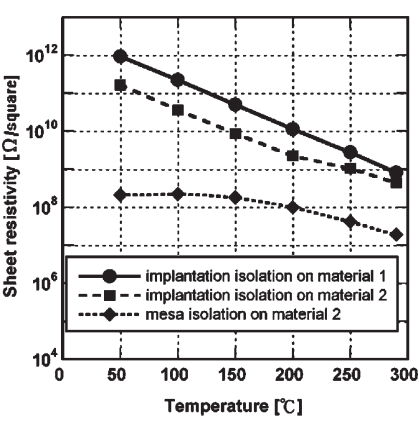

(b)
Fig. 1. (a) Sheet resistivity after 1-h annealing time versus annealing temperature. (b) Sheet resistivity versus temperature for BTS measurement at a bias of $100 \mathrm{~V}$.

in-house process described in [17] with the exception of the isolation process. The piece of material 1 and one piece of material 2 were subjected to $\mathrm{O}^{+}$ion implantation with implantation energies of 25,50 , and $75 \mathrm{keV}$, and the dose is $5 \times 10^{14} \mathrm{~cm}^{-2}$ for each energy. The TRIM software was used to simulate the implantation process [10]. On these samples, the ohmic contacts were formed before the isolation process. Another sample of material 2 was subjected to a chlorine-based inductively coupled plasma reactive ion etching mesa isolation process. The isolation test structure used in this letter consists of two $100-\mu \mathrm{m}$-wide ohmic contact with a separation of $5 \mu \mathrm{m}$.

\section{RESUlTS AND DiscusSiON}

The thermal stability of the implantation isolation was investigated in two ways: First, the sheet resistivity versus annealing temperature was measured on the isolation test structures after $1 \mathrm{~h}$ of annealing [Fig. 1(a)]. The sheet resistivity is higher than $10^{12} \Omega / \square$ up to at least $450{ }^{\circ} \mathrm{C}$ annealing. The thermal stability of the $\mathrm{O}^{+}$implantation is appropriate for subsequent HEMT processes (i.e., $\mathrm{CVD} \mathrm{SiN}_{x}$ passivation process at $300{ }^{\circ} \mathrm{C}$ ) and normal operation [18]. After $100 \mathrm{~h}$ of annealing at $300{ }^{\circ} \mathrm{C}$, the implanted material sheet resistivity was $4.3 \times 10^{12} \Omega / \square$, which is even higher compared to the sample with $1-\mathrm{h}$ annealing time. The sheet resistance of these samples was measured as a function of temperature, and in all cases, temperature-activated behavior was obtained. From this, we obtained activation energies of $0.428,0.520,0.115$, and $0.030 \mathrm{eV}$ at $300{ }^{\circ} \mathrm{C}, 450{ }^{\circ} \mathrm{C}$, $600{ }^{\circ} \mathrm{C}$, and $850{ }^{\circ} \mathrm{C}$, respectively (not shown). Second, bias temperature stress (BTS) measurements were performed on the isolation test structures at $100 \mathrm{~V}$ on the three pieces submitted to full HEMT processing [Fig. 1(b)]. All samples were held at each temperature $10 \mathrm{~min}$ before the measurement. The $\mathrm{O}^{+}$ion implantation isolation technique showed higher sheet resistivity at least up to $290{ }^{\circ} \mathrm{C}$. (The sheet resistivity in a mesa structure is the resistivity of the undoped GaN buffer.) These results show the good thermal stability of this implantation technique on samples submitted to full HEMT processing.

Fig. 2 shows the comparison of gate characteristics of the HEMTs that are fabricated on material 2 using either implantation or mesa isolation. The measurements were performed at $V_{\mathrm{ds}}=0.1 \mathrm{~V}$, and the gate bias was swept from -100 to $+4 \mathrm{~V}$.

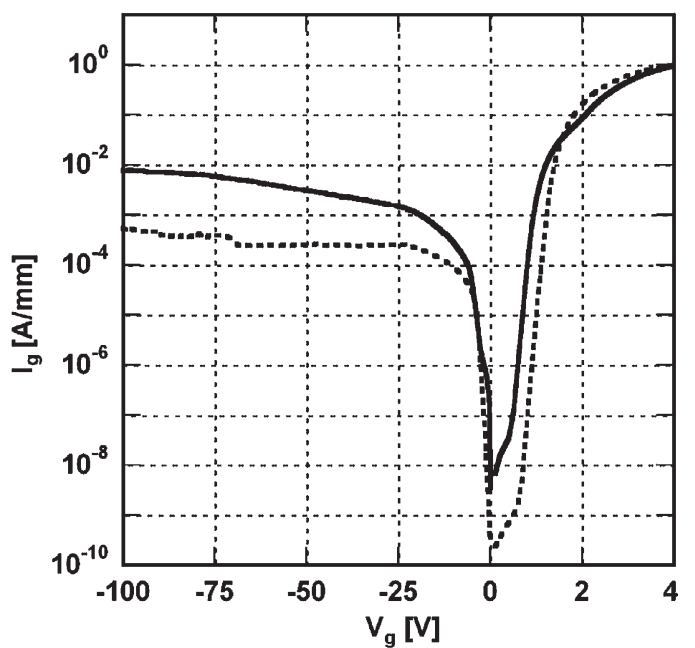

Fig. 2. Comparison of gate characteristics at $V_{\mathrm{ds}}$ of $0.1 \mathrm{~V}$ for (dotted line) oxygen ion implantation and (solid line) mesa isolation devices on material 2.

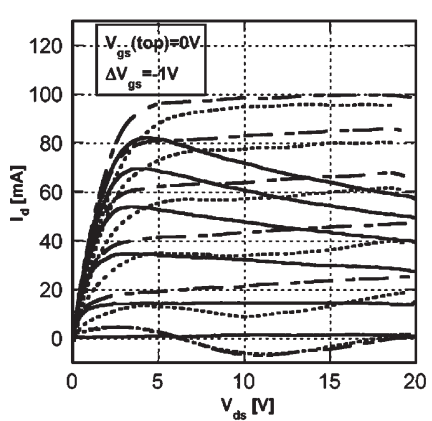

(a)

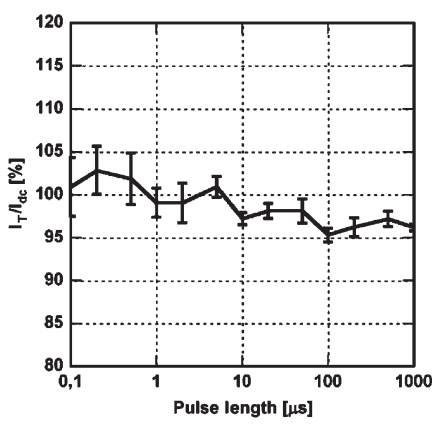

(b)
Fig. 3. (a) Transient characteristics of $\mathrm{O}^{+}$-implanted HEMTs $\left(V_{\text {gs }}\right.$ pulsed from $V_{\text {pinch }}$ to $0 \mathrm{~V}$ at $V_{\mathrm{ds}}=3 \mathrm{~V}$ ). (b) Pulsed $I-V$ characteristics from the different quiescent states (dashed line) $\left(V_{\mathrm{gs}}, V_{\mathrm{ds}}\right)(0 \mathrm{~V}, 0 \mathrm{~V})$ and (dotted line) $\left(V_{\text {pinch }}, 20 \mathrm{~V}\right)$ and (solid line) dc of $\mathrm{O}^{+}$-implanted HEMTs.

The planar implantation isolated devices have less gate-leakage current, indicating that the gate may contact the 2DEG at the sidewall of the mesa and increase the gate-leakage current [15]. The HEMTs with $2 \times 50 \times 0.6 \mu \mathrm{m}^{2}$ gates that are fabricated on material 1 shows a gate breakdown of $90 \mathrm{~V}$ at $1 \mathrm{~mA} / \mathrm{mm}$ current.

In order to investigate the trapping of electrons in the epistructures and surface, the transient and pulsed $I-V$ performance of the $\mathrm{O}^{+}$-implanted HEMTs were evaluated using a pulsed $I-V$ system (Accent DiVA D225) [17], [19]. Fig. 3(a) shows the current recovery $\left(I_{T} / I_{\mathrm{dc}}\right)$ (i.e., stepping the gate voltage from $V_{\text {pinch }}$ to $0 \mathrm{~V}$ at a given constant drain bias and monitoring the resulting transient drain current $I_{T}$ ) measurement at $V_{\mathrm{ds}}=3 \mathrm{~V}$. No gate-lag problems can be noticed for the $\mathrm{O}^{+}$-implanted HEMTs. The low $V_{\mathrm{ds}}$ is chosen to avoid complications associated with self-heating. In the literature, the presence of traps at the surface and in the AlGaN Schottky layer is used to explain the gate-lag problem seen in the gate recovery measurement [17], [20]. Comparing the dc and pulsed $I-V$ characteristics of the HEMTs, with short 100-ns pulse time and $0.1 \%$ duty cycle, is an effective way to clarify the gate-lag and drain-lag effect at the same time [17], [19]. Fig. 3(b) shows 


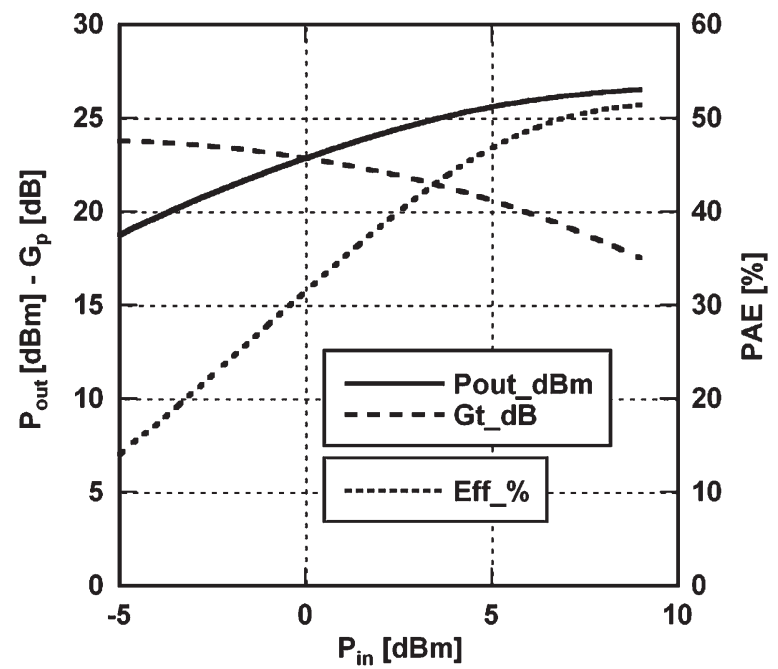

Fig. 4. Power sweep at $V_{\mathrm{ds}}=30 \mathrm{~V}$ and $V_{\mathrm{gs}}=-4 \mathrm{~V}$, showing $4.5 \mathrm{~W} / \mathrm{mm}$ saturated output power density with a PAE of $51.5 \%$.

three different measurements, namely: 1) dc; pulsed from $V_{\mathrm{gs}}=$ $0 \mathrm{~V}$ and $V_{\mathrm{ds}}=0 \mathrm{~V}$ (pulsed 1); and pulsed from $V_{\mathrm{gs}}=V_{\text {pinch }}$ and $V_{\mathrm{ds}}=20 \mathrm{~V}$ (mimicking a class B operation, pulsed 2) [19]. The pulsed 1 measurement shows higher current compared to dc due to the absence of self-heating effect. The pulsed 2 measurement shows almost no current collapse. The knee voltage in pulsed 1 and 2 measurements shows a difference of $2 \mathrm{~V}$. Based on the transient, dc, and pulsed $I-V$ measurements, we conclude that the $\mathrm{O}^{+}$-implanted isolation process does not introduce trapping problems.

The extrinsic cut-off frequency $f_{T}$ and the maximum oscillation frequency $f_{\max }$ calculated from scattering parameters measured up to $50 \mathrm{GHz}$ vector network analyzer were 33 and $57 \mathrm{GHz}$, respectively. The large signal performance of the devices was measured using continuous wave $(\mathrm{CW})$ load-pull measurements at $3 \mathrm{GHz}$ without active cooling. The saturated output power density is $4.5 \mathrm{~W} / \mathrm{mm}$ with $51.5 \%$ power added efficiency (PAE) at $V_{\mathrm{ds}}=30 \mathrm{~V}$ and $V_{\mathrm{gs}}=-4 \mathrm{~V}$ (Fig. 4). The highest output power density was $5.3 \mathrm{~W} / \mathrm{mm}$ at $V_{\mathrm{ds}}=50 \mathrm{~V}$ and $V_{\mathrm{gs}}=-4 \mathrm{~V}$.

\section{CONCLUSION}

This study has demonstrated a thermally stable electrical isolation for $\mathrm{AlGaN} / \mathrm{GaN}$ HEMT structure by multienergy implantation of oxygen ions. The ion implantation isolation process is demonstrated in the full processing of planar HEMTs, demonstrating full compatibility with HEMT processing.

The planar nature of this isolation process circumvents potential problems with enhanced gate leakage due to the gate contacting the 2DEG at the mesa sidewall.

The effect of the ion implantation isolation process on the dynamic performance of the HEMTs was investigated. The HEMTs demonstrate dispersion-free operation in dynamic measurements, which is further demonstrated by good $\mathrm{CW}$ output power and efficiency at $3 \mathrm{GHz}$. A maximum output power density of $5.3 \mathrm{~W} / \mathrm{mm}$ and a maximum PAE of $51.5 \%$ on HEMTs grown on sapphire substrate were demonstrated. Due to its good power density and efficiency without gate-lag and drain-lag problems, this planar process is a candidate for highyield GaN-based HEMT and MMIC processes.

\section{REFERENCES}

[1] U. K. Mishra, P. Parikh, and Y. F. Wu, "AlGaN/GaN HEMTs-An overview of device operation and applications," Proc. IEEE, vol. 90, no. 6, pp. 1022-1031, Jun. 2002.

[2] S. C. Binari, H. B. Dietrich, G. Kelner, L. B. Rowland, K. J. Doverspike, and D. K. Wickenden, "H, He and N implant isolation of n-type GaN," $J$. Appl. Phys., vol. 78, no. 5, pp. 3008-3011, Sep. 1995.

[3] S. J. Pearton, C. B. Vartuli, J. C. Zolper, C. Yuan, and R. A. Stall, "Ion implantation doping and isolation of GaN," Appl. Phys. Lett., vol. 67, no. 10, pp. 1435-1437, Sep. 1995

[4] R. G. Wilson, C. B. Vartuli, C. R. Abernathy, S. J. Pearton, and J. M. Zavada, "Implantation and redistribution of dopants and isolation species in GaN and related compounds," Solid State Electron., vol. 38, no. 7, pp. 1435-1437, Jul. 1995.

[5] J. C. Zolper, "Ion implantation in group III-nitride semiconductors: A tool for doping and defect studies," J. Cryst. Growth, vol. 178, no. 1/2, pp. 157-167, Jun. 1997.

[6] B. Boudart, Y. Guhel, J. C. Pesant, P. Dhamelincourt, and M. A. Poisson, "Raman characterization of $\mathrm{Ar}^{+}$ion-implanted GaN," J. Raman Spectrosc., vol. 33, no. 4, pp. 283-286, Apr. 2002.

[7] B. Boudart, Y. Guhel, J. C. Pesant, P. Dhamelincourt, and M. A. Poisson, "Raman characterization of $\mathrm{Mg}+$ ion-implanted GaN," J. Phys., Condens. Matter, vol. 16, no. 2, pp. s49-s55, Jan. 2004.

[8] T. Oishi, N. Miura, M. Suita, T. Nanjo, Y. Abe, T. Ozeki, H. Ishikawa, T. Egawa, and T. Jimbo, "Highly resistive GaN layers formed by ion implantation of $\mathrm{Zn}$ along the c-axis," J. Appl. Phys., vol. 94, no. 3, pp. 1662-1666, Aug. 2003.

[9] J. C. Zolper, A. G. Baca, and S. A. Chalmers, "Thermally stable implant isolation of p-type $\mathrm{Al}_{0.2} \mathrm{Ga}_{0.8}$ As," Appl. Phys. Lett., vol. 62, no. 20, pp. 2536-2538, May 1993.

[10] S. J. Pearton, J. C. Zolper, R. J. Shul, and F. Ren, "GaN: Processing, defects, and devices," J. Appl. Phys., vol. 94, no. 3, pp. 1662-1666, Aug. 2003.

[11] G. Dang, X. A. Cao, F. Ren, S. J. Pearton, J. Han, A. G. Baca, and R. J. Shul, "Oxygen implant isolation of $n$-GaN field-effect transistor structures," J. Vac. Sci. Technol. B, Microelectron. Process. Phenom., vol. 17, no. 5, pp. 2015-2018, Sep. 1999

[12] G. Hanington, Y. M. Hsin, Q. Z. Liu, P. M. Asbeck, S. S. Lau, M. Asif Khan, J. W. Yang, and Q. Chen, "P/He ion implant isolation technology for AlGaN/GaN HFETs," Electron. Lett., vol. 34, no. 2, pp. 193195, Jan. 1998

[13] M. Werquin, N. Vellas, Y. Guhel, D. Ducatteau, B. Boudart, J. C. Pesant, Z. Bougrioua, M. Germain, J. C. De Jaeger, and C. Gaquiere, "First results of AlGaN/GaN HEMTs on sapphire substrate using an argon-ion implantisolation technology," Microw. Opt. Technol. Lett., vol. 46, no. 4, pp. 311314, Aug. 2005.

[14] J. W. Johnson, E. L. Piner, A. Vescan, R. Therrien, P. Rajagopal, J. C. Roberts, J. D. Brown, S. Singhal, and K. J. Linthicum, "12 W/mm AlGaN-GaN HFET on silicon substrates," IEEE Electron Device Lett., vol. 25, no. 7, pp. 459-461, Jul. 2004.

[15] S. R. Bahl, M. H. Leary, and J. A. del Alamo, "Mesa-sidewall gate leakage in InAlAs/InGaAs heterostructure field effect transistors," IEEE Trans. Electron Devices, vol. 39, no. 9, pp. 2037-2043, Sep. 1992.

[16] S. R. Bahl and J. A. del Alamo, "Elimination of mesa-sidewall gate leakage in InAlAs/InGaAs heterostructures by selective sidewall recessing," IEEE Electron Device Lett., vol. 13, no. 4, pp. 195-197, Apr. 1992.

[17] V. Desmaris, M. Rudzinski, N. Rorsman, P. R. Larssen, H. Zirath, T. C. Rodle, and H. F. F. Jos, "Comparison of the dc and microwave performance of $\mathrm{AlGaN} / \mathrm{GaN}$ HEMTs grown on SiC by MOCVD with Fe doped of unintentionally doped buffer layers," IEEE Trans. Electron Devices, vol. 53, no. 9, pp. 2413-2417, Sep. 2006.

[18] J. Kuzmík, P. Javorka, A. Alam, M. Marso, M. Heuken, and P. Kordoš, "Determination of channel temperature in $\mathrm{AlGaN} / \mathrm{GaN}$ HEMTs grown on sapphire and silicon substrates using dc characterization method," IEEE Trans. Electron Devices, vol. 49, no. 8, pp. 1496-1498, Aug. 2002.

[19] R. Chu, Y. Zhou, J. Liu, D. Wang, K. J. Chen, and K. M. Lau, "AlGaNGaN double channel HEMTs," IEEE Trans. Electron Devices, vol. 52, no. 4, pp. 438-446, Apr. 2005.

[20] R. Vetury, N. Q. Zhang, S. Keller, and U. K. Mishra, "The impact of surface states on the dc and RF characteristics of AlGaN/GaN HFETs," IEEE Trans. Electron Devices, vol. 48, no. 3, pp. 560-566, Mar. 2001. 